\title{
Band-gap calculations of anisotropic phononic crystals in a square lattice
}

\author{
Feng-Lian $\mathrm{Li}^{1, \mathrm{a}}$ \\ ${ }^{1}$ School of mechanical and engineering, Beijing Information and Technology University, China \\ alifenglian@126.com
}

Keywords: band structures; anisotropic; phononic crystals

Abstract. A boundary element method is extended to calculate the band structures of anisotropic phononic crystals with different components forming the square lattice. The system may be composed of anisotropic inclusions embedded in the isotropic matrix or isotropic inclusions embedded in the anisotropic matrix. In a periodic unit cell, boundary integral equations of the matrix and the inclusion are given. Substituting the periodic boundary conditions and the interface conditions, a set of linear equations is formed. Then the relations between the wave number and the frequency are determined, which clearly exhibit the band gaps of the phononic system. Some numerical examples are used to illustrate the accuracy and efficiency of the boundary element method. Additionally, the results show that for anisotropic phononic crystal, band gaps not only depend on the periodic lattice but also the angle between the symmetry axis of orthotropic materials (different rotating angles).

\section{Introduction}

Recently, many researchers have been extended to the study of acoustic and elastic waves in periodic composites called phononic crystals $[1,2]$. The phononic crystal is an artificial crystal constructed by scatterers periodically embedded in a homogeneous host material, in which there is a contrast between the elastic constants and/or mass densities. The most interesting and important aspect of these features is that such artificial composites can exhibit elastic/acoustic wave band gaps, in which sound and vibration are all forbidden regardless of the polarization and propagating direction of the elastic/acoustic waves. The existence of absolute band gaps has been investigated both theoretically and experimentally [3, 4]. Such materials should have many potential applications such as elastic-acoustic filters, sound shields, transducers, etc. Furthermore, phononic crystals are also of practical importance in the design of vibrationless environments for high-precision mechanical systems [5].

In the past decades, several methods have been developed to compute the band structures and transmission of elastic waves propagating in phononic crystals. For example, the plane-wave expansion (PWE) method [6] was widely applied to calculate the band structures of in-plane waves propagating in solid-solid phononic crystals. But it involves the slow convergence problem and is difficult to compute the mixed fluid-solid system. Moreover, it cannot treat different interface connection conditions. Though the finite difference time domain (FDTD) method [7] and the wavelet method [8] can compute the fluid-solid system and has fast convergence, they cannot deal with the different interface connection conditions. The finite difference time domain (FDTD) method [9] considers the different modes in solids and fluids but neglects the solid-fluid interaction at the interface. Recently, the boundary element method (BEM) [10] is developed to compute the band structures of the two-dimensional phononic crystals with isotropy. In the present paper; we will extend this method to the anisotropic phononic crystals.

\section{Theory}

We consider a 2D phononic crystal consisting of a square with the lattice constant a, see Fig. 1. Both scatterers and matrix are homogeneous, anisotropic and linear elastic solids. The governing equations describing these time-harmonic wave motions can be expressed as [11] 


$$
\left\{\Gamma_{i j}\left(\partial_{1}, \partial_{2}\right)+\rho \omega^{2} \delta_{i j}\right\} u_{j}(\mathbf{x})=0,
$$

where $u_{j}(\mathbf{x})$ is the component of the displacement; $\rho$ is the mass density; $\omega$ is the angular frequency.

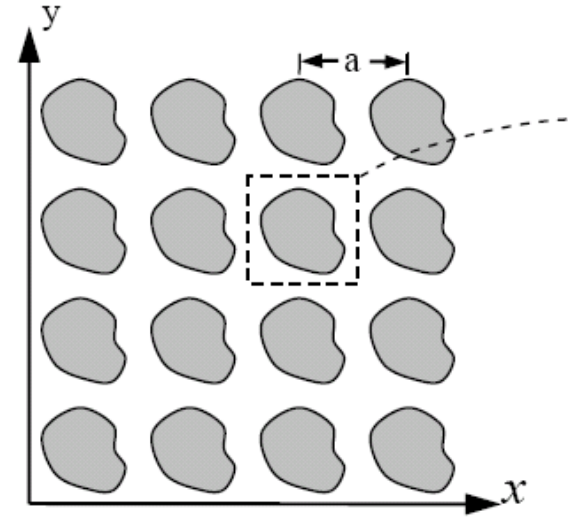

(a)

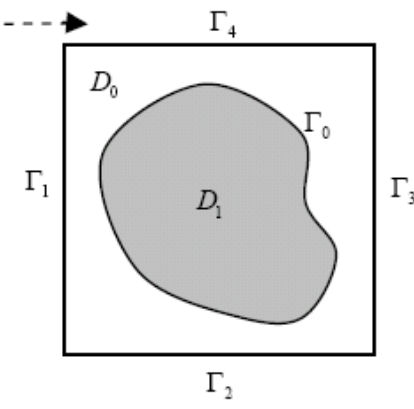

(b)

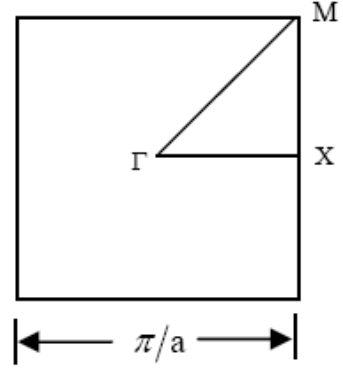

(c)

Fig. 1. The structures under consideration: (a), (b) and (c) are the square lattice, its corresponding square unit cell and the first Brillouin zone, respectively.

Due to the periodicity of the system, we can restrict our attention to a unit cell (Fig. 1 (a2).The boundary or interface conditions for the problem are the continuities of the displacement field and the stress field on the interface $\Gamma_{0}$ between the matrix and the scatterers. Thus we have the following relations

$$
\mathbf{u}_{1}(\mathbf{r})=\mathbf{u}_{0}(\mathbf{r}), \quad \mathbf{T}_{1}(\mathbf{r})=\mathbf{T}_{0}(\mathbf{r}), \quad \mathbf{r} \in \Gamma_{0},
$$

where $\mathbf{T}_{1}(\mathbf{r})$ and $\mathbf{T}_{0}(\mathbf{r})$ are the traction vectors.

In addition, the periodicity of the structures implies that all field quantities (e.g. the displacements, stresses and their derivatives) should satisfy the Bloch theorem. Accordingly, the displacement vector $\mathbf{u}$ can be written as

$$
\mathbf{u}\left(\mathbf{r}+\mathbf{r}_{m}\right)=e^{i \mathbf{k} \cdot \mathbf{r}_{m}} \mathbf{u}_{\mathbf{k}}(\mathbf{r}),
$$

where $\mathbf{k}=\left(k_{x}, k_{y}\right)$ is a real Bloch wave vector and $\mathbf{u}_{\mathbf{k}}(\mathbf{r})$ follows the same periodic condition as the structure; $i=\sqrt{-1}$; and $\mathbf{r}_{m}=m_{1} \mathbf{a}_{1}+m_{2} \mathbf{a}_{2}$ with $\left(m_{1}, m_{2}\right) \in Z^{2}$ and $\mathbf{a}_{1}$ and $\mathbf{a}_{2}$ being the fundamental translation vectors of the lattices.

According to the reciprocal theorem, the boundary integral equation for the transverse wave can be expressed as [11]

$$
c u_{z}\left(\mathbf{x}_{Q}\right)+\int_{\Gamma} \frac{\partial u_{z}^{*}}{\partial \mathbf{n}}\left(\mathbf{x}_{Q} ; \mathbf{x}_{P}\right) \cdot u_{z}\left(\mathbf{x}_{P}\right) d \Gamma_{P}=\int_{\Gamma} u_{z}^{*}\left(\mathbf{x}_{Q} ; \mathbf{x}_{P}\right) \cdot \frac{\partial u_{z}}{\partial \mathbf{n}}\left(\mathbf{x}_{P}\right) d \Gamma_{P},
$$

where $\Gamma$ is the boundary; $c=0.5$ for a smooth boundary; and $\mathbf{x}_{Q}$ and $\mathbf{x}_{P}$ are the field and source points, respectively. In Eq. (4) the fundamental solution or Green's function is given by the document[11]. The detailed process can be referenced in the literature [10].

\section{Numerical results}

Based on the above described theory, some typical phononic crystal systems are calculated in this section for the transverse wave modes. The accuracy and the efficiency of the method are compared with other methods, and the effects of the anisotropy of the material on the band gaps are discussed. 
Now, we consider the cylinders ybco with orthorhombic symmetry embedded in the epoxy matrix with isotropy forming a square lattice. The filling ratio is $f=0.75$. The density and the elastic parameters of the component materials are: $\rho_{0}=1142 \mathrm{~kg} / \mathrm{m}^{3}, c_{11}=7.54 \mathrm{Gpa}, c_{12}=4.57 \mathrm{Gpa}$ and $c_{44}=1.48 \mathrm{Gpa}$ for epoxy; and $\rho_{1}=6333 \mathrm{~kg} / \mathrm{m}^{3}, c_{11}^{1}=268 \mathrm{Gpa}, c_{12}^{1}=132 \mathrm{Gpa}, c_{13}^{1}=95 \mathrm{Gpa}, c_{22}^{1}=231 \mathrm{Gpa}, c_{23}^{1}=71 \mathrm{Gpa}$, $c_{33}^{1}=186 \mathrm{Gpa}, c_{44}^{1}=37 \mathrm{Gpa}, c_{55}^{1}=49 \mathrm{Gpa}$ and $c_{66}^{1}=95 \mathrm{Gpa}$ for ybco. We choose 8 constant elements on each edge of the external boundary and 32 constant elements on the boundary of the scatterer. The band structures for the square lattices are plotted in Fig. 2. It can be observed that in the calculation frequency interval, there is one complete band gap between the first and the second band, and between the fourth and the fifth band, respectively, namely [0.786, 1.254], and [2.596, 2.752]. These results are in agreement with the results computed by the plane wave expansion method [12, 13]. It verifies that the present method is accurate and effective. Additionally, in the present method, the forming matrix is smaller and the computing time is less.

In the above system, the transverse wave mode is related to the elastic parameters $c_{44}, c_{45}$ and $c_{55}$. And these elastic constants are dependent upon the rotating angles. Additionally, $c_{44}$ and $c_{55}$ have larger difference, so the rotating angles have larger influence on the bang gaps. Table 1 lists the range of the band gaps with the different rotating angles. We can find that the width of the first band gap become bigger, then decrease with the rotating angle increasing in the lower frequency, but the width of the second band gap becomes smaller, then increase with the rotating angle increasing in the higher frequency. Generally, the orthotropic scatterers have effect on the band gaps.

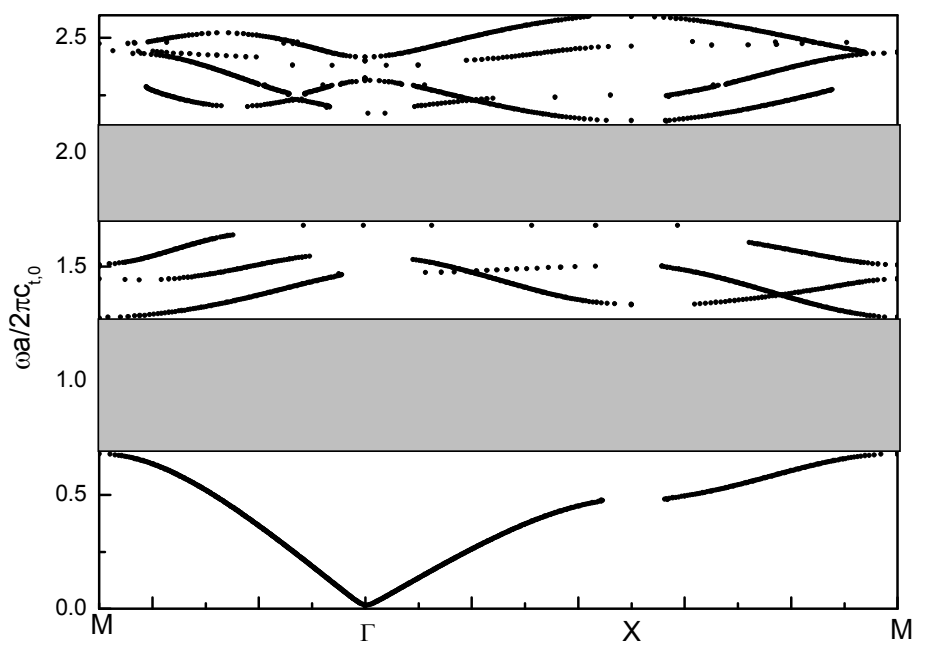

Fig.2 Band structures for the ybco/epoxy system in a square with filling fraction $f=0.75$ and the rotation angle $\theta=0^{\circ}$

Table 1 the band gaps for ybco/epoxy with the different rotation angles $\left(\omega a / 2 \pi c_{t, \text { epoxy }}\right)$

\begin{tabular}{|l|c|c|c|l|l|l|}
\hline Rotating angle & $\theta=0^{0}$ & $\theta=15^{0}$ & $\theta=30^{0}$ & $\theta=45^{0}$ & $\theta=60^{0}$ & $\theta=90^{0}$ \\
\hline The first band gap & {$[0.786,1.254]$} & {$[0.660,1.260]$} & {$[0.660,1.258]$} & {$[0.660,1.255]$} & {$[0.660,1.240]$} & {$[0.660,1.200]$} \\
\hline $\begin{array}{l}\text { The second band } \\
\text { gap }\end{array}$ & {$[1.680,2.136]$} & {$[1.685,2.125]$} & {$[1.685,2.115]$} & {$[1.685,2.115]$} & {$[1.685,2.130]$} & {$[1.685,2.150]$} \\
\hline
\end{tabular}

\section{Conclusions}

In this paper the boundary element method is extended to compute the band structures of anisotropic phononic crystals. The influence of rotating orthotropic scatterers on the wave band gaps is investigated numerically in the case of the square lattice. It is verified that the present method is accurate and effective for the anisotropic phononic crystals. In addition, the computed results show that the transverse wave mode is affected by rotating scatterers. Therefore, the location and width of 
complete stop bands can be adjusted by changing the rotating angle of anisotropic scatterers. In summary, the wave band structure of 2D phononic crystal with anisotropic scatterers can be designed elaborately with consideration of the anisotropic feature of scatterers except the lattice, the material constant contrast, the filling fraction and the interface conditions.

\section{Acknowledgements}

This work was financially supported by the National Natural Science Foundation of China (Grant Nos. 11202021, 11472249) and the Postdoctoral Science Foundation of China (2012M510311).

\section{References}

[1] M. S. Kushwaha, and P. Halevi. Appl. Phys. Lett. 64 (1994), p. 1085.

[2] J. Mei, C. Qiu, and Z. Liu. J. Phys-condens. Mat. 17(2005), p.3735.

[3] P. Lambin, A. Khelif, J. O. Vasseur, L. Dobrzynski, and B. Djafari-Rouhani. Phys. Rev. E 63(2001), p. 066605.

[4] Z. Liu, C. T. Chan, P. Sheng, A. L. Goertzen, and J. H. Page. Phys. Rev. B 62(2000), p. 2446.

[5] F.C. Hsu, T.T. Wu, J.C. Hsu, J.H. Sun. Appl Phys Lett 93(2008), P. 201904.

[6] M. S. Kushwaha, P. Halevi. Appl. Phys. Lett. 69 (1996), P. 31.

[7] Y. Tanaka, Y. Tomoyasu, and S. Tamura. Phys. Rev. B 62(2000), P. 7387.

[8] Z.Z. Yan, Y.S. Wang, and Ch. Zhang. Acta Mech. Solida Sin. 21(2008), P. 104.

[9] Y. J. Cao, Z. L. Hou, Y. Y. Liu. Solid State Commun. 132 (2004), p. 539.

[10] F.L. Li, Y.S. Wang, Ch. Zhang, and G.L. Yu. Eng. Anal. Bound. Elem. 37(2013), P.225.

[11] Ch. Zhang, D. Gross D. Computational Mechanics Publications, Southampton, 1998.

[12] Z.Q. Zhan, P.J. Wei. Acta Mech. Solida Sinica 23 (2010), P. 181.

[13] T.T. Wu, Z.G. Huang, and S. Lin. Phys. Rev. B 69 (2004), P. 094301. 